\title{
Gla-rich Protein (GRP): A New Player in the Burden of Vascular Calcification
}

Carla SB Viegas and Dina C Simes*

${ }^{1}$ Centre of Marine Sciences (CCMAR), University of Algarve, Portugal

${ }^{2}$ GenoGla Diagnostics, Centre of Marine Sciences (CCMAR), University of Algarve, Faro, Portugal

*Corresponding author: Simes DC, Centre of Marine Sciences (CCMAR), University of Algarve, Campus de Gambelas, Portugal, Tel: +351 289800100 ; Fax: +351 289 800069; E-mail: dsimes@ualg.pt

Rec date: May 13, 2016; Acc date: June 17, 2016; Pub date: June 22, 2016

Copyright: ( $) 2016$ Viegas CSB, et al. This is an open-access article distributed under the terms of the Creative Commons Attribution License, which permits unrestricted use, distribution, and reproduction in any medium, provided the original author and source are credited.

\begin{abstract}
Vascular calcification is a life-threatening complication of cardiovascular disease and an independent risk factor for morbidity and mortality. It is a complex and active cellular process that includes multiple players and regulators resembling physiological bone formation. Currently, it is generally accepted that our understanding of the molecular aetiology of vascular calcification remains incomplete. Endogenous inhibitors play a critical role in preventing vascular calcification. A relation between vitamin $\mathrm{K}$ and vascular calcification has been widely established and recently, the vitamin K-dependent protein, Gla-rich protein (GRP), was proposed to have an important role in vascular calcification inhibition, with clinical implications.
\end{abstract}

Keywords: Gla-rich protein; Vascular calcification; Calcification inhibitors; Vitamin K

\section{Introduction}

Vascular calcification (VC) is an active and regulated multifactorial process highly relevant in the general population and particularly in advanced age, atherosclerosis, diabetes and chronic kidney disease $[1,2]$. Essential steps in the pathological calcification pathway involve vascular smooth muscle cells (VSMCs) osteochondrogenic differentiation, with up-regulation of bone mineralization-regulatory genes, and spontaneous release of extracellular vesicles (EVs), resembling developmental bone formation [3-6]. Although many aspects concerning the pathogenesis of $\mathrm{VC}$ are still unclear, multiple factors, such as proliferation and differentiation of resident VSMCs, loss of anticalcific mechanisms, release of calcifying EVs, VSMCs apoptosis, endothelial dysfunction, oxidative stress, increased extracellular matrix (ECM) remodeling, and chronic inflammation, contribute to the development and progression of calcific lesions $[2-4,7,8]$. Under physiological conditions, local and systemic inhibitors of mineral formation act to prevent widespread tissue calcification influencing osteochondrogenic differentiation, formation of calcifying competent EVs, and ECM crystal growth [2,5,9]. In fact, several in vivo and in vitro studies shown that this is a naturally occurring process that must be actively inhibited since the presence of certain inhibitors are crucial to prevent pathological calcification [9-12]. In the last decades, many molecules have been demonstrated to act as mineralization inhibitors, such as the vitamin K-dependent matrix gla protein (MGP), fetuin-A (or alpha 2-Heremans-Schmid glycoprotein, AHSG), osteopontin (OPN), pyrophosphate, bone sialoprotein, and osteoprotegerin, among others. The discovery of additional mineralization inhibitors, as is the case of Gla-rich protein (GRP), will uncover new molecular pathways and contribute to understand the interaction between calcification and inflammation processes occurring in VC.

\section{GRP and Vascular Calcification}

GRP is the newest member of the vitamin K-dependent protein (VKDP) family, first identified in sturgeon calcified cartilage [13]. Gla residues within VKDPs result from post-translation modification of specific glutamic acid residues (Glu) by the $\gamma$-glutamyl carboxylase, using vitamin $\mathrm{K}$ as cofactor, and conferring high calcium-binding properties [14-16]. Fully $\gamma$-carboxylated GRP can include an unprecedented number of $\gamma$-carboxyglutamic acid (Gla) residues (15 Gla residues in human) [13]. Continuous recycling of the active form of vitamin $\mathrm{K}$ is performed by vitamin $\mathrm{K}$ epoxide reductase, which is inhibited by coumarin-derived drugs (e.g. warfarin), acting as vitamin $\mathrm{K}$ antagonists and impairing the $\gamma$-carboxylation of VKDPs [14-17]. A relation between vitamin $\mathrm{K}$ and vascular calcification has been widely established and several factors, such as insufficient intake of vitamin $\mathrm{K}$, mutations in the $\gamma$-carboxylase enzyme, and warfarin treatment, leading to reduced or abolished $\gamma$-carboxylation of VKDPs, have been shown to induce arterial calcifications $[15,18-20]$. The action of vitamin $\mathrm{K}$ in arterial calcification has been mostly associated with MGP function that, until recently, was considered to be the central Gla protein involved in the prevention of soft tissue calcification and local mineralization of the vascular wall [21,22]. Interestingly, while MGP knockout mice die within 8 weeks of birth due to massive vascular mineralization [21], loss-of-function mutations in the human MGP gene, known as the Keutel syndrome, result in non-lethal abnormal soft tissue calcifications [23], suggesting that additional or compensatory mechanisms of mineralization inhibition might exist in human. The unprecedented high density of Gla residues of GRP, conferring an outstanding capacity for calcium-binding, together with its wide pattern of tissue distribution/accumulation in mammals and high evolutionary conservation in vertebrates, have suggested a critical function as global calcium modulator [13,24]. Its calcium-binding properties and association with calcification processes have been demonstrated through immunohistochemical and in vitro studies showing high levels of protein accumulation at sites of pathological calcification [24-27], and the capacity to directly bind basic calcium phosphate (BCP) crystals [26]. GRP has been proposed as a negative regulator of osteogenic differentiation [28], a modulator of calcium 
availability in the ECM $[13,24]$, and as an inhibitor of calcification in the cardiovascular and articular systems [27,29]. Increased levels of calcification inhibitors at sites of calcification, such as MGP, have been associated with increased accumulation of the non-functional uncarboxylated protein form (ucMGP) and related to a deficient $\gamma$ carboxylation system [15,19,22]. Similarly, increased levels of undercarboxylated GRP (ucGRP) was found associated with pathological calcification-related diseases, as calcified aortic valve stenosis [27], osteoarthritis [25] and certain cancers [26]. The activity of VKDPs depends on their $\gamma$-carboxylation status, and non- or undercarboxylated protein forms are often regarded as non-functional. This has been widely demonstrated for MGP, whose $\gamma$-carboxylation is an absolute requirement for its calcification-inhibitory activity $[15,19,22]$.
In the case of GRP, although both $\gamma$-carboxylated (cGRP) and ucGRP protein forms have mineral-binding affinity, only cGRP was shown to be able to inhibit calcification in the vascular and articular systems [27,29]. Under calcification stimuli, increased expression of VKDPs such as GRP and MGP might function to counteract calcification, but system overload can lead to exhaustion of vitamin $\mathrm{K}$ storage hampering $\gamma$-carboxylation capacity, with consequent increase in nonfunctional undercarboxylated protein forms. It should be pointed that functional studies on GRP showed contradictory results, emphasizing that differences exist between animal models [30,31], and specific efforts should be undertaken to clearly establish the molecular mechanism of GRP action in human (Figure 1).

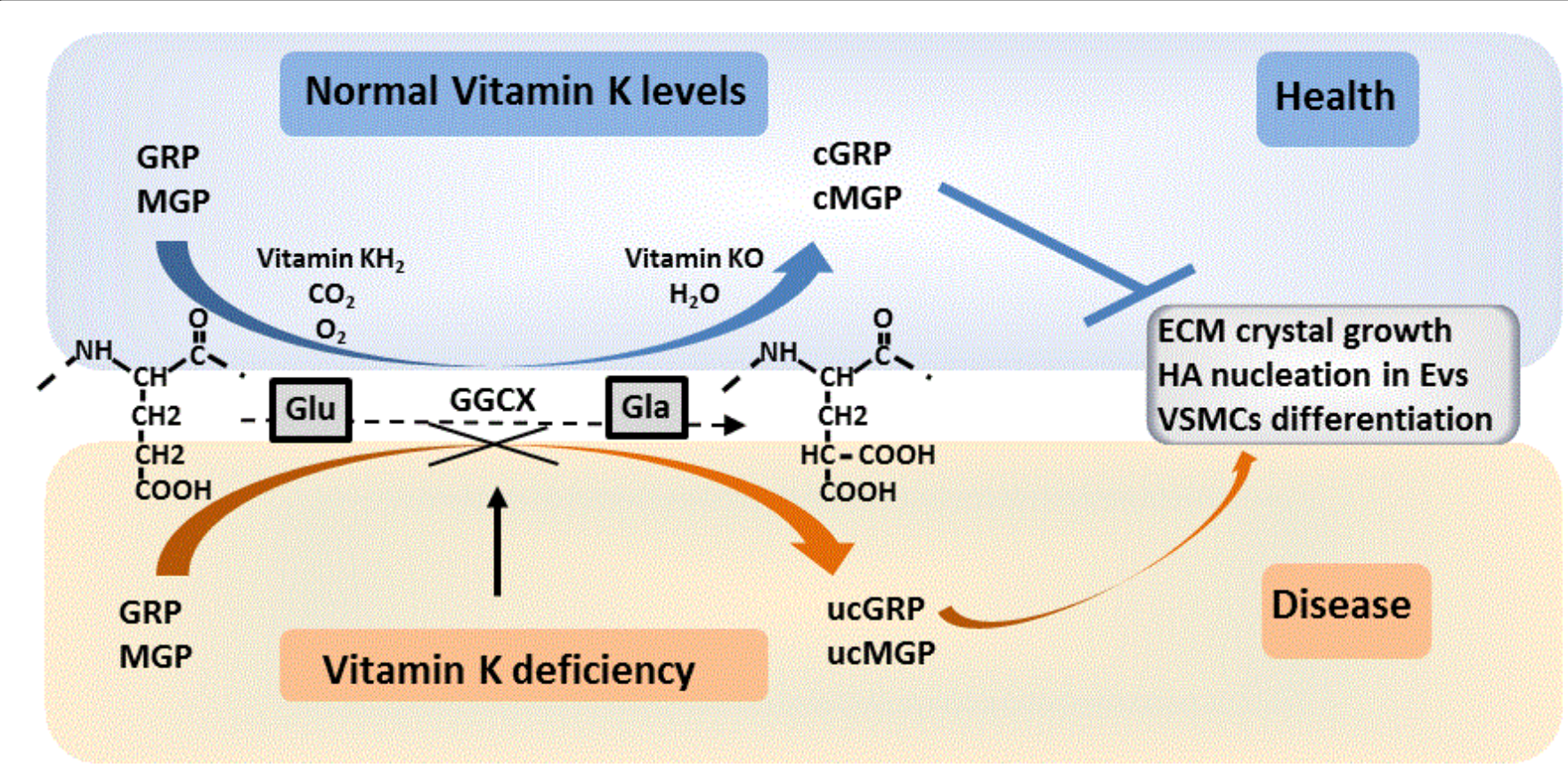

Figure 1: Vitamin $\mathrm{K}$ and vascular calcification. In healthy conditions, the levels of vitamin $\mathrm{K}$ are sufficient to support $\gamma$-carboxylation of MGP and GRP, where specific glutamic acid residues (Glu) are converted to $\gamma$-carboxyglutamic acid residues (Gla). This $\gamma$-carboxylation reaction is catalyzed by the $\gamma$-glutamyl carboxylase (GGCX) enzyme and requires the presence of a reduced form of vitamin K (vitamin KH2), carbon dioxide and oxygen, resulting in the formation of Gla residues and the epoxide form of vitamin $\mathrm{K}$ (vitamin $\mathrm{KO}$ ). $\gamma$-carboxylated GRP and MGP (cGRP and cMGP) are capable to inhibit vascular mineralization by modulation of crystal growth in the extracellular matrix (ECM), hydroxyapatite (HA) nucleation in EVs and VSMCs differentiation. In vitamin K deficiency conditions, $\gamma$-carboxylation of GRP and MGP are impaired, resulting in the accumulation of undercarboxylated protein forms (ucGRP and ucMGP) lacking calcification inhibitory capacity and consequently promoting vascular mineralization. VKAs, vitamin K antagonists.

VSMCs have a key role in the initiation and regulation of pathological VC. The plasticity of VSMCs allow their osteogenic conversion in the presence of osteogenic signals, with consequent changes from contractile to synthetic physiological roles, characterized by loss of smooth muscle lineage markers such as alpha smooth muscle actin ( SSMA), SM22 alpha (SM22 $\alpha$ ), and increased expression of genes known to be master players in osteogenesis including bone morphogenetic proteins (BMPs), runt-related transcription factor 2
(Runx2 or Cbfa-1), osterix, osteocalcin (OC), and alkaline phosphatase, among others [1-3,11]. This VSMCs osteochondrogenic differentiation was shown to be inhibited by the addition of exogenous cGRP, which was able to up-regulate aSMA while down-regulated osteopontin (OPN) expression, retaining VSMCs in a contractile phenotype [27]. Furthermore, GRP was found to be present in VSMCs-derived EVs and associated with its mineralization competence (Figure 2). 

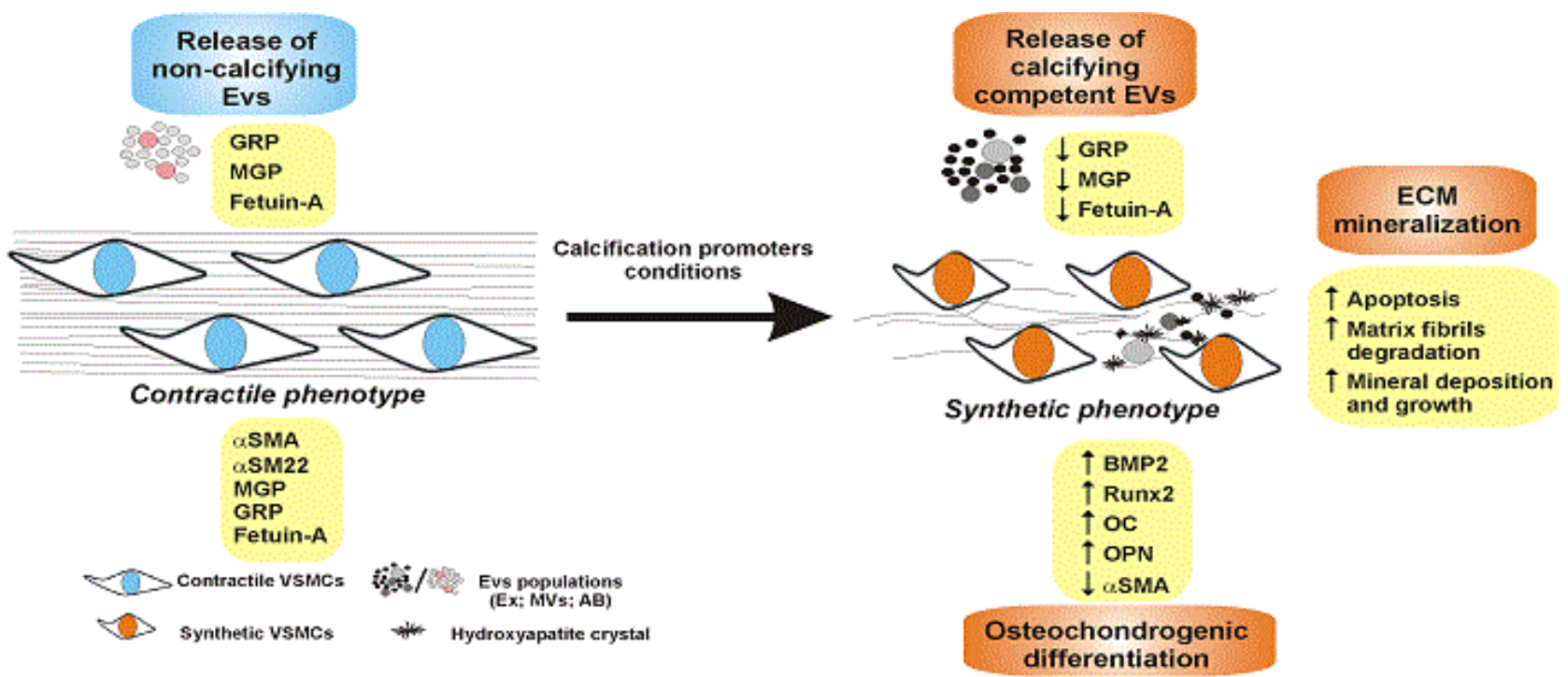

Figure 2: Proposed mechanism for vascular calcification. Under physiological conditions VSMCs maintain a contractile phenotype by the expression of contractile apparatus proteins (aSM-22, aSMA), production of mineralization inhibitors (MGP, GRP), uptake of fetuin-A and release of extracellular vesicles (EVs) loaded with mineralization inhibitors. Under calcification promoters conditions, such as uremia, inflammatory and oxidative stress, vitamin $\mathrm{K}$ deficiency, endothelial dysfunction, and aging, VSMCs down regulate the expression of contractile-associated genes and up-regulate the expression of several osteogenic markers (BMP-2, Runx2, OC, OPN) acquiring a synthetic phenotype. These events culminate on the osteochondrogenic differentiation of VSMCs and the release of calcifying EVs, characterized by a decreased cargo of mineralization inhibitors and hydroxyapatite nucleation crystals. Increased bone-associated proteins and the deposition of calcifying competent EVs ultimately leads to extracellular matrix mineralization and degradation, and increased cell apoptosis. Ex: exosomes, MVs, matrix vesicles, $\mathrm{AB}$, apoptotic bodies, ECM, extracelular matrix.

It is known that vascular calcification is a process that can be initiated with the deposition in the ECM of BCP-containing EVs derived from VSMCs, forming a nidus for calcification in a process similar to bone mineralization [4-6]. Under normal conditions, VSMCs-derived EVs do not calcify due to their loading with mineralization inhibitors. Calcifying EVs with increased calcium loading are known to have decreased MGP, fetuin-A, and more recently also shown to be depleted of GRP. This supports the theory that loss of mineralization inhibitors to block mineral nucleation is crucial to increase EVs mineralization competence capable of accelerating ECM calcification. Interestingly, GRP was found to be part of an MGP-fetuin-A protein complex at sites of valvular calcification [27]. Although the presence of a similar protein complex within EVs is still unknown, the association of these three proteins, with high calcification-inhibitory capacity, might represent a powerful antimineralization system. The notion that EVs-mediated mineralization occurring in pathological and physiological processes share many similarities, and that EVs content can regulate mineralization, has fostered an increased attention to the role of EVs in the field of ectopic mineralization of connective tissues. The unique features of these secreted membrane-enclosed vesicles, containing functional proteins and RNA species with the capacity of transfer cargo to other cells, is currently changing our view of intercellular communication mechanisms, with possible implications for disease progression $[5,32,33]$. Their potentialities as a novel source of disease biomarkers and drug-delivery systems applied to cardiovascular calcification might represent an exciting new therapeutic approach. However, important questions mostly related to the biogenesis, assemblage and secretion regulatory mechanisms, and to the EVs capacity of affecting neighboring or distant target cells and ECM, are still to be resolved in the cardiovascular calcification field. Atherosclerosis is an example of the complex interplay between multicellular-derived EVs affecting atherosclerotic plaques. These EVs are heterogeneous in size and mineral content, and may originate from VSMCs, endothelial cells, or leukocytes (e.g. macrophages, lymphocytes and granulocytes) [34]. It was recently shown that CD36 mediate the effect of diverse sources of EVs in endothelial cells, and to be required for foam cells formation with a preponderant role in atherosclerosis [35,36]. Considering a possible CD36-EVs mediated pathway, it is likely that the contribution to atherosclerosis of additional cell-derived EVs, including circulatingEVs, is still to be uncovered. Macrophages were shown to release calcifying-EVs loaded with mineralization-related factors capable of accelerate ECM calcification [37]. It is currently accepted that in calcification-related chronic inflammatory diseases, such as atherosclerosis, calcification and inflammation are involved in a complex interplay with several crosstalk factors in a positive feed-back loop that drives disease progression. Activated macrophages produce soluble factors that promote osteochondrogenic differentiation of VSMCs [38-39] and release calcifying EVs that might directly contribute to mineral deposition [37]. Also, micro-calcifications have been proposed to be involved in macrophage recruitment in early stages of atherosclerosis [40], and BCP crystals reported to stimulate macrophages to produce pro-inflammatory cytokines affecting VSMCs differentiation [41]. GRP was recently shown to be involved in the crosstalk between inflammation and calcification of articular tissues in osteoarthritis, acting as an anti-inflammatory agent [29]. In addition to VSMCs and valvular interstitial cells, GRP was found accumulated in foam cells at atheromatous areas of calcified aortic valves [27]. Further 
highlights on the role of GRP in calcification and inflammation processes, its involvement with inflammatory cells, and its regulation in EVs loading are warranted, and might become highly relevant to increase our current knowledge on the pathophysiological mechanisms of cardiovascular calcification and possible therapeutical approaches.

\section{Conclusion}

Although many questions are still open concerning its molecular mechanisms of action, GRP has been demonstrated as a key factor involved in cardiovascular calcification, representing a potential therapeutic target for the inhibition of vascular calcification by functional modulation through vitamin K. Simultaneously, this information reinforces the notion that special care should be given to the clinical use of anticoagulant coumarin-derived drugs for prevention and long term treatment of thromboembolic disorders, since they act as vitamin $\mathrm{K}$ antagonists, interfering with MGP and GRP functionality and representing an important drawback for this therapy.

\section{Acknowledgements}

This work was funded by projects PTDC/SAU-ORG/117266/2010 and PTDC/BIM-MEC/1168/2012, and also through project UID/ Multi/04326/2013, all from the Portuguese Science and Technology Foundation (FCT). CSB Viegas was the recipient of the FCT fellowship SFRH/BPD/70277/2010.

\section{References}

1. Demer LL, Tintut Y (2013) Vascular calcification: pathobiology of a multifaceted disease. Circulation 117: 2938-2948.

2. Paloian NJ, Giachelli CM (2014) A current understanding of vascular calcification in CKD. Am J Physiol Renal Physiol 307: F891-900.

3. Sage AP, Tintut Y, Demer LL (2010) Regulatory mechanisms in vascular calcification. Nat Rev Cardiol 7: 528-536

4. Kapustin AN, Chatrou ML, Drozdov I, Zheng Y, Davidson SM, et al (2015) Vascular smooth muscle cell calcification is mediated by regulated exosome secretion. Circ Res 116: 1312-1323.

5. New SE, Aikawa E (2013) Role of extracellular vesicles in de novo mineralization: an additional novel mechanism of cardiovascular calcification. Arterioscler Thromb Vasc Biol 33: 1753-1758.

6. Cui L, Houston DA, Farquharson C, MacRae VE (2016) Characterisation of matrix vesicles in skeletal and soft tissue mineralisation. Bone 87: 147-158.

7. Doherty TM, Asotra K, Fitzpatrick LA, Qiao JH, Wilkin DJ, et al. (2003) Calcification in atherosclerosis: bone biology and chronic inflammation at the arterial crossroads. Proc Natl Acad Sci USA 100: 11201-11206.

8. Aikawa E, Nahrendorf M, Figueiredo JL, Swirski FK, Shtatland T, et al. (2007) Osteogenesis associates with inflammation in early-stage atherosclerosis evaluated by molecular imaging invivo. Circulation 116: 2841-2850.

9. Jahnen-Dechent W, Heiss A, Schäfer C, Ketteler M (2011) fetuin-A regulation of calcified matrix metabolism. Circ Res 108: 1494-1509.

10. Schinke T, Karsenty G (2000) Vascular calcification--a passive process in need of inhibitors. Nephrol Dial Transplant 15: 1272-1274.

11. Wallin R, Wajih N, Greenwood GT, Sane DC (2001) Arterial calcification: a review of mechanisms, animal models, and the prospects for therapy. Med Res Rev 21: 274-301.

12. Tsang HG, Rashdan NA, Whitelaw CB, Corcoran BM, Summers KM, et al. (2016) Large animal models of cardiovascular disease. Cell Biochem Funct 34: 113-132.

13. Viegas CS, Simes DC, Laizé V, Williamson MK, Price PA, et al. (2008) Gla-rich protein (GRP), a new vitamin K-dependent protein identified from sturgeon cartilage and highly conserved in vertebrates. J Biol Chem 283: 36655-36664.

14. Tie JK, Stafford DW (2016) Structural and functional insights into enzymes of the vitamin K cycle. J Thromb Haemost 14: 236-247.

15. Willems BA, Vermeer C, Reutelingsperger CP, Schurgers LJ (2014) The realm of vitamin $\mathrm{K}$ dependent proteins: shifting from coagulation toward calcification. Mol Nutr Food Res 58: 1620-1635.

16. Cranenburg EC, Schurgers LJ, Vermeer C (2007) Vitamin K: the coagulation vitamin that became omnipotent. Thromb Haemost 98: 120-125.

17. van Gorp RH, Schurgers LJ (2015) New Insights into the Pros and Cons of the Clinical Use of Vitamin K Antagonists (VKAs) Versus Direct Oral Anticoagulants (DOACs). Nutrients 7: 9538-9557.

18. Price PA, Faus SA, Williamson MK (1998) Warfarin causes rapid calcification of the elastic lamellae in rat arteries and heart valves. Arterioscler Thromb Vasc Biol 18: 1400-1407.

19. Schurgers LJ, Spronk HM, Soute BA, Schiffers PM, DeMey JG, et al (2007) Regression of warfarin-induced medial elastocalcinosis by high intake of vitamin $\mathrm{K}$ in rats. Blood 109: 2823-2831.

20. Krüger T, Oelenberg S, Kaesler N, Schurgers LJ, van de Sandt AM, et al. (2013) Warfarin induces cardiovascular damage in mice. Arterioscler Thromb Vasc Biol 33: 2618-2624.

21. Luo G, Ducy P, McKee MD, Pinero GJ, Loyer E, et al. (1997) Spontaneous calcification of arteries and cartilage in mice lacking matrix GLA protein. Nature 386: 78-81.

22. Schurgers LJ, Uitto J, Reutelingsperger CP (2013) Vitamin K-dependent carboxylation of matrix Gla-protein: a crucial switch to control ectopic mineralization. Trends Mol Med 19: 217-226.

23. Munroe PB, Olgunturk RO, Fryns JP, Van Maldergem L, Ziereisen F, et al. (1999) Mutations in the gene encoding the human matrix Gla protein cause Keutel syndrome. Nat Genet 21: 142-144.

24. Viegas CSB, Cavaco S, Neves PL, Ferreira A, João A, et al. (2009) Gla-rich protein (GRP) is a novel vitamin $\mathrm{K}$ dependent protein present in serum and accumulated at sites of pathological calcifications. Am J Pathol 175: 2288-2298.

25. Rafael MS, Cavaco S, Viegas CSB, Santos S, Ramos A, et al. (2014) Insights into the association of Gla-rich protein and osteoarthritis, novel splice variants and $\gamma$-carboxylation status. Mol Nutr Food Res 58: 1636-1646.

26. Viegas CS, Herfs M, Rafael MS, Enriquez JL, Teixeira A, et al. (2014) Glarich protein is a potential new vitamin $\mathrm{K}$ target in cancer: evidences for a direct GRP-mineral interaction. Biomed Res Int 2014: 340216.

27. Viegas CSB, Rafael M, Enriquez JL, Teixeira A, Vitorino R, et al. (2015) Gla-rich protein (GRP) acts as a calcification inhibitor in the human cardiovascular system. Arterioscler Thromb Vasc Biol 35: 399-408.

28. Surmann-Schmitt C, Dietz U, Kireva T, Adam N, Park J, et al. (2008) Ucma, a novel secreted cartilage-specific protein with implications in osteogenesis. J Biol Chem 283: 7082-7093.

29. Cavaco S, Viegas CS, Rafael MS, Ramos A, , et al. (2016) Gla-rich protein is involved in the cross-talk between calcification and inflammation in osteoarthritis. Cell Mol Life Sci 73: 1051-1065.

30. Eitzinger N, Surmann-Schmitt C, Bosl M, Schett G, Engelke K, et al. (2012) Ucma is not necessary for normal development of the mouse skeleton. Bone 50: 670-680.

31. Neacsu CD, Grosch M, Tejada M, Winterpacht A, Paulsson M, et al. (2011) Ucmaa (Grp-2) is required for zebrafish skeletal development. Evidence for a functional role of its glutamate $\gamma$-carboxylation. Matrix Biol 30: 369-378.

32. Kapustin AN, Shanahan CM (2016) Emerging roles for vascular smooth muscle cell exosomes in calcification and coagulation. J Physiol 594: 2905-2914.

33. Krohn JB, Hutcheson JD, Martinez-Martinez E, Aikawa E (2016) Extracellular vesicles in cardiovascular calcification: expanding current paradigms. J Physiol 594: 2895-2903. 
Citation: Viegas CSB, Simes DC (2016) Gla-rich Protein (GRP): A New Player in the Burden of Vascular Calcification. J Cardiovasc Dis Diagn 4: 245. doi:10.4172/2329-9517.1000245

Page 5 of 5

34. Leroyer AS, Isobe H, Lesèche G, Castier Y, Wassef M, et al. (2007) Cellular origins and thrombogenic activity of microparticles isolated from human atherosclerotic plaques. J Am Coll Cardiol 49: 772-777.

35. Ramakrishnan DP, Hajj-Ali RA, Chen Y, Silverstein RL (2016) Extracellular Vesicles Activate a CD36-Dependent Signaling Pathway to Inhibit Microvascular Endothelial Cell Migration and Tube Formation. Arterioscler Thromb Vasc Biol 36: 534-544.

36. Chen Y, Kennedy DJ, Ramakrishnan DP, Yang M, Huang W, et al. (2015) Oxidized LDL-bound CD36 recruits an Naâo/Kâa-ATPase-Lyn complex in macrophages that promotes atherosclerosis. Sci Signal 8: 91.

37. New SE, Goettsch C, Aikawa M, Marchini JF, Shibasaki M, et al. (2013) Macrophage-derived matrix vesicles: an alternative novel mechanism for microcalcification in atherosclerotic plaques. Circ Res 113: 72-77.

38. Tintut Y, Patel J, Territo M, Saini T, Parhami F, et al. (2002) Monocyte/ macrophage regulation of vascular calcification in vitro. Circulation 105: 650-655.
39. Ikeda K, Souma Y, Akakabe Y, Kitamura Y, Matsuo K, et al. (2012) Macrophages play a unique role in the plaque calcification by enhancing the osteogenic signals exerted by vascular smooth muscle cells. Biochem Biophys Res Commun 425: 39-44.

40. Chatrou ML, Cleutjens JP, van der Vusse GJ, Roijers RB, Mutsaers PH, et al. (2015) Intra-Section Analysis of Human Coronary Arteries Reveals a Potential Role for Micro-Calcifications in Macrophage Recruitment in the Early Stage of Atherosclerosis. PLoS One 10: e0142335.

41. Nadra I, Mason JC, Philippidis P, Florey O, Smythe CD, et al. (2005) Proinflammatory activation of macrophages by basic calcium phosphate crystals via proteinkinase C and MAP kinase pathways: a vicious cycle of inflammation and arterial calcification? Circ Res 96: 1248-1256. 